\title{
Laboratory measurements to interpret dust polarimetric observations and in-situ radar studies. Implications for the Rosetta mission.
}

\author{
A.-Ch. Levasseur-Regourd ${ }^{1}$, Yann Brouet ${ }^{2}$ and Edith Hadamcik ${ }^{1}$ \\ ${ }^{1}$ UPMC - LATMOS - CNRS, Sorbonne Univ., BC 102, 4 place Jussieu, 75005 Paris, France \\ email: aclr@latmos.ipsl.fr \\ ${ }^{2}$ Physics Institute, University of Bern, Sidlerstrasse 5, 3012 Bern, Switzerland \\ email: yann.brouet@space. unibe.ch
}

\section{Context}

Polarimetric astronomical observations on dust clouds and regolithic surfaces require laboratory simulations on samples to provide clues to properties of the scattering media. Similarly, in-situ radar investigations of Solar System bodies require laboratory studies to infer the physical properties of their interiors. Recent developments are illustrated by analyses of comet $67 \mathrm{P} /$ Churyumov-Gerasimeko (C-G) remote observations and in-situ studies from Rosetta mission.

\section{Interpretation of polarimetric observations and local radar studies}

The polarization of solar light scattered by dust clouds depends on dust properties. The PROGRA2 suite of photo-polarimetric instruments (in the visible to near IR domains) has been developed to document the properties of dust samples studied in microgravity conditions or on surfaces (e.g., Levasseur-Regourd et al. 2015). Satisfactory fits for remote observations of C-G at its 2008-2009 passage were derived for mixtures of aggregates and compact particles, with an absorbing component (Hadamcik et al. 2010). The presence of large dust particles and of refractory organics in the pre-perihelion coma is now established by Rosetta results (e.g., Schulz et al. 2015).

Radar absorption inside a planetary body depends on the length of the propagation path and on its complex permittivity. Measurements of the permittivity of various porous dust samples (of 45 to $65 \%$ porosity) have been developed to constrain the properties of the subsurface and interior of C-G, as explored by MIRO on Rosetta and CONSERT on Philae (Brouet et al. 2015). While CONSERT at $90 \mathrm{MHz}$ has measured a real part of the permittivity about 1.27 (Kofman et al. 2015), our measurements suggest this value to correspond to a porosity of $80 \%$ for a dust/ice volumetric ratio of 0.3 . For $1.27 \pm 0.05$, the porosity could be about 80 to $85 \%$, with a dust/ice ratio within 0.1 to 1.5 , in fair agreement with interpretations derived by Kofman et al. (2015) from an other approach.

\section{References}

Brouet, Y., Levasseur-Regourd, A. C., Sabouroux, P. et al. 2015, Astron. Astrophys., in press Hadamcik, E., Sen, A. K., Levasseur-Regourd, A. C. et al. 2010, Astron. Astrophys., 517, A86 Kofman, W., Herique, A., Barbin, Y., Barriot, J. P., Ciarletti, V. et al. 2015, Science, 349, 6247 Levasseur-Regourd, A. C., Renard, J. B., Shkuratov, Y. et al. 2015, in: L. Kolokolova et al. (eds.), Polarimetry of Stars and Planetary Systems (Cambridge University Press), p. 62 Schulz, R., Hilchenbach, M., Langevin, Y., Kissel, J., Silen, J. et al. 2015, Nature, 518, 216 